\title{
Adsorption of synthetic homo- and hetero-oligodeoxynucleotides onto highly oriented pyrolytic graphite: Atomic force microscopy characterization
}

\author{
Ana-Maria Chiorcea Paquim ${ }^{a}$, Tatiana S. Oretskaya ${ }^{b}$, Ana Maria Oliveira Brett ${ }^{\mathrm{a}, *}$ \\ a Departamento de Química, Faculdade de Ciências e Tecnologia, Universidade de Coimbra, 3004-535 Coimbra, Portugal \\ ${ }^{\mathrm{b}}$ Chemistry Department, Moscow State University, Vorobjovy Gory, 119899, Moscow, Russia
}

Received 14 September 2005; received in revised form 22 December 2005; accepted 22 December 2005

Available online 7 February 2006

\begin{abstract}
DNA adsorption on electrode surfaces is of fundamental interest for the development of DNA-based biosensors. The free adsorption of 10-mer synthetic oligodeoxynucleotides (ODNs) onto highly oriented pyrolytic graphite (HOPG) surfaces was studied using Magnetic AC mode atomic force microscopy (MAC Mode AFM). The mechanism of interaction of nucleic acids with carbon electrode surfaces was elucidated, using 10-mer synthetic homo- and hetero-ODNs sequences of known base sequences, because they allow clear interpretation of the experimental data. AFM images in air revealed different adsorption patterns and degree of HOPG surface coverage for the ODNs, and correlation with the individual structure and base sequence of each ODN molecule will be presented. The results demonstrated that the hydrophobic interactions with the HOPG hydrophobic surface explain the main adsorption mechanism, although other effects such as electrostatic and Van der Waals interactions may contribute to the free adsorption process. The ODNs interacted differently with the HOPG surface, according to the ODN sequence hydrophobic characteristics, being directly depending on the molecular mass, the hydrophobic character of the individual bases and on the secondary structure of the molecule. The importance of the type of base existent at the ODN chain extremities on the adsorption process was investigated and different adsorption patterns were obtained with ODN sequences composed by the same group of bases aligned in a different order.
\end{abstract}

(C) 2006 Elsevier B.V. All rights reserved.

Keywords: AFM; Oligodeoxynucleotides; ODN; Adsorption; DNA-biosensor; Hydrophobic interactions

\section{Introduction}

Deoxyribonucleic acid (DNA) is a very important biomolecule that plays a crucial role in all living organisms, being responsible for storage, duplication, and genetic information [1]. Due to its important chemical and biophysical characteristics, that establish a high specificity of recognition and binding to other molecules, recently nanotechnology and biosensor technology were examining the DNA molecules for construction of DNA-based biosensor devices [2-6]. In particular, DNA electrochemical biosensors have received a special attention due to numerous successful applications in medicine, environmental and food control [4-9].

A DNA-electrochemical biosensor consists of an electrochemical transducer (the electrode) with an immobilized nucleic

* Corresponding author. Tel./fax: +351 239835295.
E-mail address: brett@ci.uc.pt (A.M. Oliveira Brett). acid film on its surface (the probe). Depending on the required application, the control of DNA adsorption is an essential step for the design and construction of efficient DNA-based electrochemical biosensors. Many factors can influence the efficiency of DNA immobilization on the electrode and on the nature of DNA-surface interactions: the electrode characteristics and electrode pre-treatment conditions, the DNA adsorption procedure, the $\mathrm{pH}$ and ionic strength of the DNA solution, the sequence, length and concentration of the DNA molecules. Many of the biophysical properties of DNA and its flexibility are influenced by these factors. Therefore it is important to have a good understanding of all key factors that influence the immobilization of the nucleic acid probe onto the transducer surface.

In recent years, atomic force microscopy (AFM) has proved to be a powerful technique to investigate the interfacial and conformational proprieties of biological samples [10-12]. AFM can visualize individual DNA molecules and DNA-protein 
complexes immobilized onto different surface materials, with extraordinary resolution and accuracy.

Special attention must be paid to investigate DNA molecules immobilized on the conducting surfaces of electrochemical transducers. Magnetic AC mode AFM (MAC Mode AFM) permits investigation of DNA molecules weakly bound to the electrodes. MAC Mode AFM was used to study long chain calfthymus double-stranded DNA (dsDNA) and single-stranded DNA (ssDNA) molecules immobilized onto highly oriented pyrolytic graphite (HOPG) electrode surfaces [13-16]. Both ssDNA and dsDNA molecules spontaneously self-assembled forming two-dimensional lattices with uniform coverage of the HOPG surface. The DNA network patterns formed during adsorption of long chain DNA molecules are very important for DNA-electrochemical biosensors for detection of DNA-drug interactions, since nucleic acid lattices define nanoelectrode systems with different active surface areas on the graphite substrate.

The nature of the DNA interactions with carbon electrode surfaces, as well as the topographical conformations that short length DNA molecules can adopt are still not clearly understood. However the specific mechanism of interaction of nucleic acid molecules with the electrode surface is difficult to establish using long chain molecules. Short chain 10-mer oligodeoxynucleotides (ODNs) molecules, with various specific sequences, represent particularly attractive models to study nucleic acid adsorption because they allow a clear interpretation of the experimental data.

Immobilization of short single-stranded ODNs at the electrode surface is also essential for the development of DNA-biosensors for hybridization detection. The hybridization strategy at a DNA-electrochemical biosensor consists of two

\section{purine bases}<smiles>Nc1nc2[nH]cnc2c(=O)[nH]1</smiles>

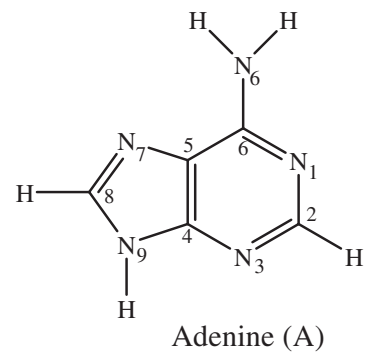

pyrimidine bases<smiles></smiles>

Cytosine (C)<smiles>Cc1c[nH]c(=O)[nH]c1=O</smiles>

Thymine (T)
Fig. 1. Chemical structure of DNA bases. steps: immobilization of the ODN sequence (the probe) on the electrode surface and hybridization with the complementary strand (target) present in solution. In this context, the electrode surface coverage, orientation and packing of the adsorbed ODN probe layer has to be determined. Imaging small adsorbed ODNs can provide valuable information about the morphology adopted by these molecules at the electrode surface.

Therefore, a systematic MAC Mode AFM study at room temperature of the adsorption and conformational changes of different ODN molecules onto HOPG surface was carried out, in order to elucidate the mechanism of binding of short sequence DNA molecules at carbon electrode surfaces.

The ODN chain is formed by monomeric nucleotide units, each one being composed of three types of chemical components, a phosphate group, a deoxyribose and four different nitrogen bases: adenine (A) and guanine $(\mathrm{G})$, the purine bases, cytosine $(\mathrm{C})$ and thymine $(\mathrm{T})$, the pyrimidine bases, Fig. 1. In this study homo-ODNs, composed only of one type of base in the sequence, and hetero-ODNs, formed by a mixture of different bases, were both used.

The results revealed an interesting correlation between the degree of surface coverage and the base sequence of the ODN molecules, which will help in elucidating the mechanism of the interaction of nucleic acids with carbon electrode surfaces.

\section{Experimental section}

\subsection{Materials}

The ODNs used were synthesized on an Applied Biosystems 380B automated DNA synthesizer (USA), using reagents for ODN chemistry purchased from Fluka (Germany). The purity of the ODN sequences was verified by NMR and HPLC analysis. The base sequences were:
A10
G10
$\mathrm{C} 10$
$\mathrm{T} 10$
A6G4
A8G2
$\mathrm{T} 8 \mathrm{C} 2$
$\mathrm{T} 6 \mathrm{C} 4$
MIX 1
MIX 2

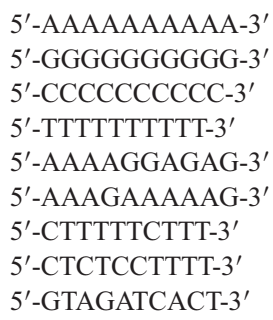

To simplify the interpretation of the AFM data, the abbreviations related for the base sequence of each ODN molecule will be used in the article. It is also worth noting that sequences A10 is complementary to the sequence T10, G10 to C10, A6G4 to T6C4, A8G2 to T8C2, and MIX 1 to MIX 2.

Microvolumes were measured using EP-10 and EP-100 Plus Motorized Microliter Pipettes (Rainin Instruments Co. Inc., Woburn, USA). The $\mathrm{pH}$ measurements were carried out with a GLP 21 Crison $\mathrm{pH}$ meter.

The electrolyte used was $\mathrm{pH} \quad 4.5 \quad 0.1 \mathrm{M}$ acetate buffer solution and was prepared using analytical grade reagents and purified water from a Millipore Milli-Q system (conductivity $<0.1 \mu \mathrm{S} \mathrm{cm}{ }^{-1}$ ). Solutions of different concentrations 
were obtained by direct dilution of the appropriate volume in acetate buffer.

HOPG, grade ZYB, of rectangular shape with $15 \times 15 \times 2 \mathrm{~mm}$ dimensions, from Advanced Ceramics Co., UK/Germany, was used throughout this study as a substrate. The HOPG was freshly cleaved with adhesive tape prior to each experiment and was imaged by AFM in order to establish its cleanliness.

\subsection{Sample preparation}

The ODN samples were prepared by free adsorption. $100 \mu \mathrm{L}$ samples of $0.3 \mu \mathrm{M}$ ODN solutions were deposited onto freshly cleaved HOPG surfaces and incubated for $3 \mathrm{~min}$. The excess of ODN was gently cleaned with a jet of Millipore Milli-Q water, and the HOPG with adsorbed ODN was then dried with nitrogen.

\subsection{Atomic force microscopy}

AFM was performed with a PicoSPM controlled by a MAC Mode module and interfaced with a PicoScan controller from Molecular Imaging Corp., Tempe, AZ. All the AFM experiments were performed with a CS AFM S scanner with the scan range $6 \mu \mathrm{m}$ in $x-y$ and $2 \mu \mathrm{m}$ in $z$ (Molecular Imaging Corp.). Silicon type II MAClevers of $225 \mu \mathrm{m}$ length, tip radius of curvature less than $10 \mathrm{~nm}, 2.8 \mathrm{~N} \mathrm{~m}^{-1}$ spring constant, and 60 $90 \mathrm{kHz}$ resonant frequencies in air (Molecular Imaging Corp.) were used in MAC Mode AFM. All images (256 samples line ${ }^{-1} \times 256$ lines) were taken at room temperature, with scan rates of 1.0-2.5 lines $\mathrm{s}^{-1}$. The images were processed by flattening in order to remove the background slope, and the contrast and brightness were adjusted. All images were visualized in three dimensions using the Scanning Probe Image Processor, SPIP, version 3.3, Image Metrology ApS, Denmark.

\subsection{Statistics}

Section analysis over ODN molecules and films was performed with PicoScan software version 5.3.1, Molecular Imaging $\mathrm{Co}$ and with Origin version 6.0 from Microcal Software, Inc., USA. The mean values of the heights were calculated using 50 measurements over different scanned images. Origin version 6.0 from Microcal Software, Inc., USA, was used to calculate standard deviation and all the experimental height/thickness distribution graphs.

\section{Results and discussion}

The ODN base composition strongly influences the observed morphology of ODN adsorption onto an HOPG surface. From the AFM experimental results a relationship between the degree of surface coverage and the individual structure of each molecule was determined and the mechanism of interaction of the ODN molecules with the surface will be discussed.

Several DNA immobilization methods have been described in the literature, but DNA spontaneous adsorption is the simplest, since it does not require any modification of the

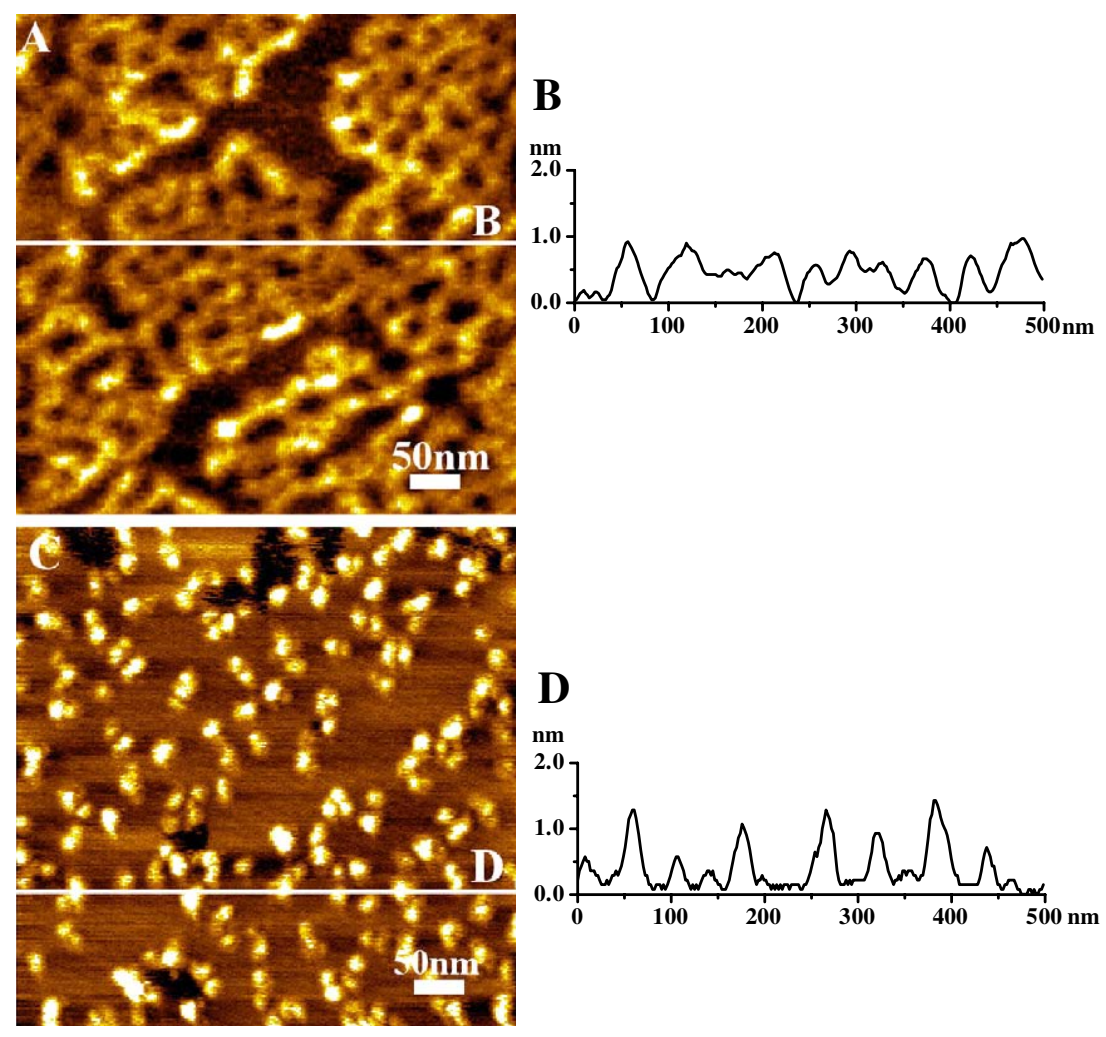

Fig. 2. (A, C) MAC Mode AFM topographical images in air of ODN molecules, immobilized onto HOPG by free adsorption during 3 min, from $0.3 \mu \mathrm{M}$ (A) A10 and (C) G10, in pH 4.5 0.1 M acetate buffer. (B, D) Cross-section profiles through white lines in images (A) and (C). 
nucleic acid molecules or of the surface. The HOPG electrode was modified by free adsorption with thin films of the 10-mer synthetic ODNs and, in all the experiments, solutions of $0.3 \mu \mathrm{M}$ ODNs in $0.1 \mathrm{M}$ acetate buffer $\mathrm{pH} 4.5$ were used, as described in the Experimental section. A pH of 4.5 was chosen, since it has been used in many electrochemical applications with DNAelectrochemical biosensors [6].

However, free adsorption was performed for different deposition times ( $30 \mathrm{~s}, 3 \mathrm{~min}, 10 \mathrm{~min}$ and, respectively, $25 \mathrm{~min}$ ) and different ODN concentrations $(0.1,0.3$ and $0.8 \mu \mathrm{M})$. The time of $3 \mathrm{~min}$ and $0.3 \mu \mathrm{M}$ solution concentration were chosen in order to obtain an incomplete coverage of the surface for most of the ODN sequences. This adsorption procedure allowed a valid comparison between the adsorption pattern of different molecules, while for higher adsorption times and ODN concentrations the adsorption reaches the saturation and the AFM images showed dry uniform films covering the electrode completely, with insignificant differences between the different ODN sequences.

\subsection{Adsorption of homo-ODNs onto $H O P G$}

The inherent capacity of different ODN base sequences to adsorb specifically on the HOPG surface is difficult to explain using ODNs of mixed base sequences. Homo-ODNs, containing residues of identical nucleotides, enable comparison of the morphological properties of each individual base. Therefore the adsorption of A10, G10, C10 and T10, all four 10-mer homoODNs, by MAC Mode AFM in air, was investigated.

Topographical images in air of the HOPG electrode modified by a thin film of A10 molecules showed that the molecules adsorb spontaneously onto HOPG and form an incomplete network on the surface, Fig. 2A. The net was composed of small molecules adsorbed side by side on the surface, which formed close-packed "polymer like" chains. The film had many pores, the dark regions in the images, with exposed HOPG surface at the bottom of the pores. The measured thickness of the A10 layer was calculated from cross section analysis as being $1.0 \pm 0.3 \mathrm{~nm}$, Fig. 2B.

In the AFM images the G10 molecules looked like small globular aggregates, well dispersed on the HOPG, Fig. 2C. The degree of surface coverage was much smaller than in the case of A10 molecules. The average height and standard deviation of the spherical aggregates was $1.1 \pm 0.3 \mathrm{~nm}$, Fig. 2D, higher than the values obtained for the A10 network.

Similar results have been obtained with $\mathrm{C} 10$ molecules, the degree of HOPG coverage being even less, Fig. 3A. The average height of the spherical $\mathrm{C} 10$ molecules measured was $0.9 \pm 0.3$ nm, Fig. 3B.

Images of adsorption onto HOPG from T10 solution showed a non-compact thin network, Fig. 3C. The small molecules of T10 adsorb onto the HOPG surface next to each other, forming looped filaments with large portions of uncovered HOPG
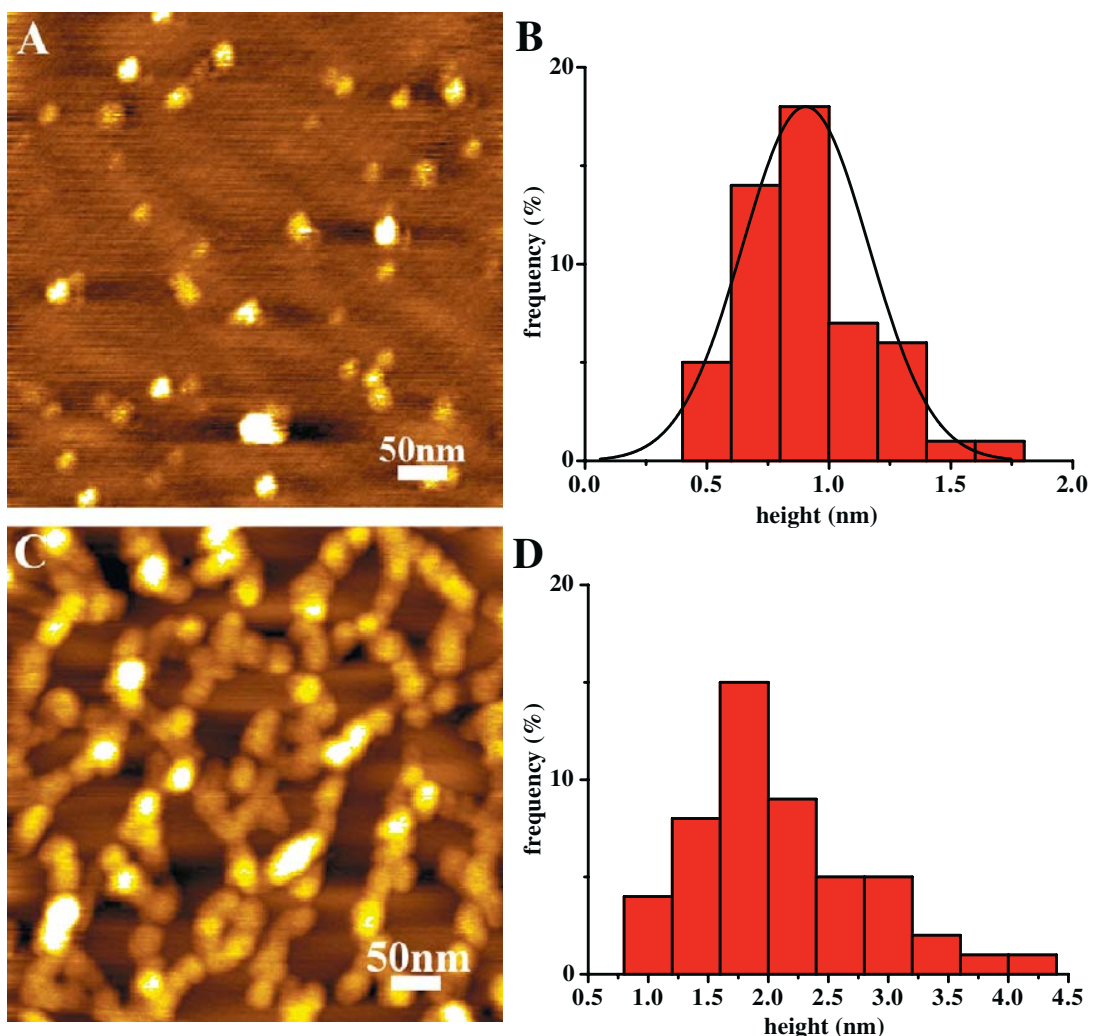

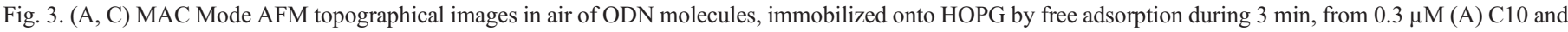

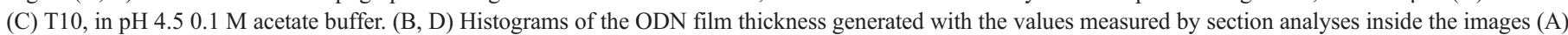
and $(\mathrm{C})$. 
surface. This layer presented a smaller degree of coverage when compared with the A10 molecules, Fig. 2A, demonstrating a smaller interaction with the HOPG. The measured thickness of the T10 film was very irregular, with heights between 0.8 and $4.5 \mathrm{~nm}$, as observed on the height distribution graph from Fig. 3D.

The 10-mer ODN molecules attached to the HOPG surface are very small. Assuming that highly dehydrated singlestranded DNA adopts the A-form after drying on the substrate, we estimate the average dimensions of a standard ODN molecule to be approximately $2.6 \mathrm{~nm}$ helix diameter and approximately $0.28 \mathrm{~nm}$ average axial rise per base, which for a standard 10-mer ODN molecule gives an average length of $2.8 \mathrm{~nm}$.

The geometric parameters of the AFM tip represent a limiting factor in the high resolution approach to investigation of biological materials. For such small molecules, the width of ODN molecules measured from the AFM images is usually much larger than the real ODN helix diameter or length, due to the convolution effect of the tip radius.

Rough estimates for the AFM measured width of adsorbed molecules can be obtained using a simplified model of parabolic or spherical tip in contact with a small, well-defined, incompressible, spherical molecule. In the case of an AFM tip with spherical geometry, the apparent width, $l$, of a molecule with molecular radius $r$ depends on the AFM tip radius $R$ according to the formula $l=\sqrt{R r}$ [17], while for an AFM tip with a parabolic geometry and $R>r$, the apparent width is $l=$

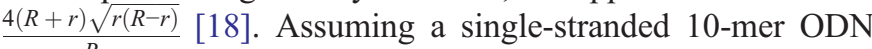
molecule is a sphere, for a tip radius of approximately $10 \mathrm{~nm}$ we expect an apparent width of the ODN molecule of $21.2 \mathrm{~nm}$ for a spherical and $23.0 \mathrm{~nm}$ for a parabolic AFM tip geometry. Consequently, a single ODN molecule will appear in the AFM images as a spherical aggregate, with overestimated helix diameter and apparent length, due to the convolution effect of the AFM tip radius of curvature.

Indeed, the small 10-mer G10 and C10 molecules appear in the AFM images as small globular aggregates with a measured full width at half-maximum height (fwhm) of approximately 10-30 nm. Nevertheless, since the tip is responsible for such a big contribution to the measured width, it is not possible to distinguish between one and two molecules that condensate together.

On the other hand, during the sample scan, the AFM tip geometry has a dynamic behavior due to the adsorption of sample onto the tip and breakage of small parts of the tip, incidents that can lead the increase of the tip radius of curvature. Additionally, the ODNs are soft and easily deformed, which results in an increased fwhm of adsorbed ODN observed in the images.

Comparing with the fwhm measurements, the height give a better representation of the ODN diameter, since it is not limited by the tip radius of curvature. In AFM studies in air, the reported heights for DNA attached to a solid substrate vary from 0.5 to $1.9 \mathrm{~nm}$ [12]. The difference between the true DNA height and the measured one is due to elastic deformations to DNA caused by the AFM tip.
Consequently we can conclude that the heights of approximately $0.8-1.1 \mathrm{~nm}$ of the A10 and T10 networks and G10, C10 aggregates correspond to a monolayer of molecules. The large measured height of the T10 layer is due to superposition and the formation of several layers onto HOPG.

\subsection{Adsorption of hetero-ODNs onto $H O P G$}

The investigation of 10-mer ODNs with mixed base sequences proved to be a more complex situation.

The adsorption process onto HOPG of ODNs containing only purinic bases was investigated using A8G2 and A6G4 molecules.

The AFM image of A6G4 sequences freely adsorbed onto HOPG demonstrated that the molecules self-organize in a very tight and well spread two-dimensional network, presenting only a few visible pores, Fig. 4A and B, and an almost complete HOPG surface coverage, Fig. 4C. The measured thickness of the lattice also had a large spectrum of values, with heights between 0.4 and $4.0 \mathrm{~nm}$, Fig. 4D.

Adsorption onto HOPG from A8G2 solution showed the molecules also forming a compact network, Fig. 5A and B. The A8G2 presented a slightly decreased HOPG coverage than A6G4 molecules. The layer shows many holes of $1.4 \pm 0.3 \mathrm{~nm}$ in depth, Fig. 5C, leading to exposed HOPG surface at the bottom of the holes. The height distribution graph showed more regular values, Fig. $5 \mathrm{C}$, compared with the results previously obtained with A6G4 molecules.

The effect of adsorption onto HOPG of ODNs containing only pyrimidinic bases was assessed using T8C2 and T6C4 molecules, and the surface characteristics were analyzed.

The T8C2 sequence has a predominance of thymines and a cytosine at one extremity of the strand. The adsorption pattern of T8C2 molecules, Fig. 6A-C, was similar to that of T10 homoODNs, Fig. 3C. The measured thickness of the aggregated fibers forming the network was irregular, with heights between 0.7 and $3.0 \mathrm{~nm}$, Fig. 6D, due to the initiation of multilayer formation. Sporadically globular aggregates of height up to $5 \mathrm{~nm}$ could be measured. The T8G2 and A8G2 molecules are complementary base sequences. As observed in the AFM images, the T8C2 sequences presented a smaller degree of surface coverage, Fig. 6 , when compared with the degree of HOPG surface coverage of A8G2 sequences, Fig. 5.

When the number of cytosines in the chain was increased, T6C4, the adsorption was radically different. The MAC Mode AFM images showed only a few globular aggregates stacked on the surface, Fig. 7A, with heterogeneous heights between 0.9 and $3.0 \mathrm{~nm}$, Fig 7B. The adsorption of T6C4 molecules presented a completely different adsorption pattern on the HOPG surface, than its complementary sequence A6G4, Fig. 4 , with a higher degree of surface coverage.

Finally, the adsorption of two ODN sequences containing the same mixture of all DNA bases in a different order was analyzed. The two ODN mixed sequences presented a complementary structure.

The MIX 1 molecules assembled forming an almost complete coverage of the HOPG surface, Fig. 8A. The 

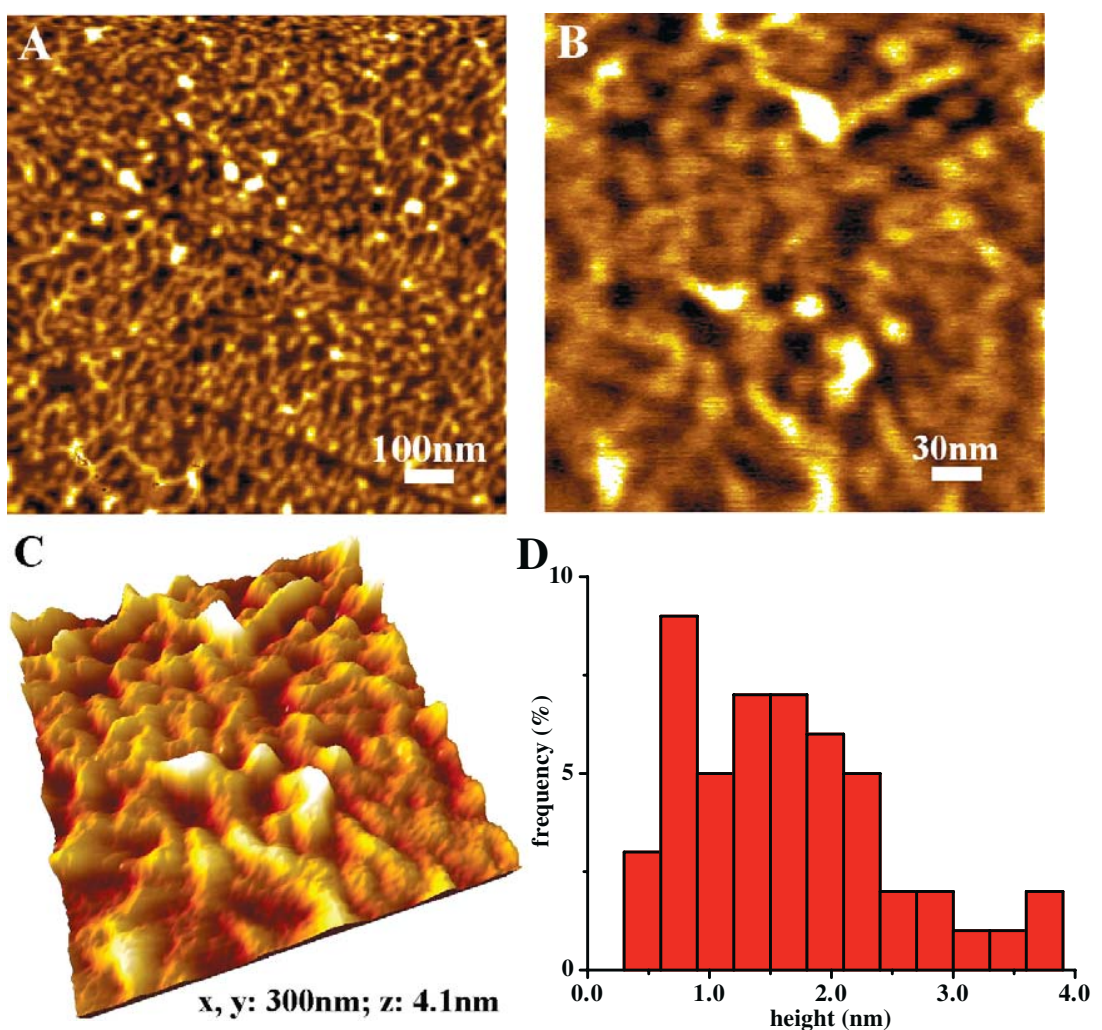

Fig. 4. (A, B) MAC Mode AFM topographical images in air of A6G4 molecules, immobilized onto HOPG by free adsorption during 3 min, from $0.3 \mu \mathrm{M}$ A6G4, in pH 4.5 0.1 M acetate buffer. (C) Three-dimensional representation of image (B). (D) Histogram of the A6G4 film thickness generated with the values measured by section analyses inside the images.
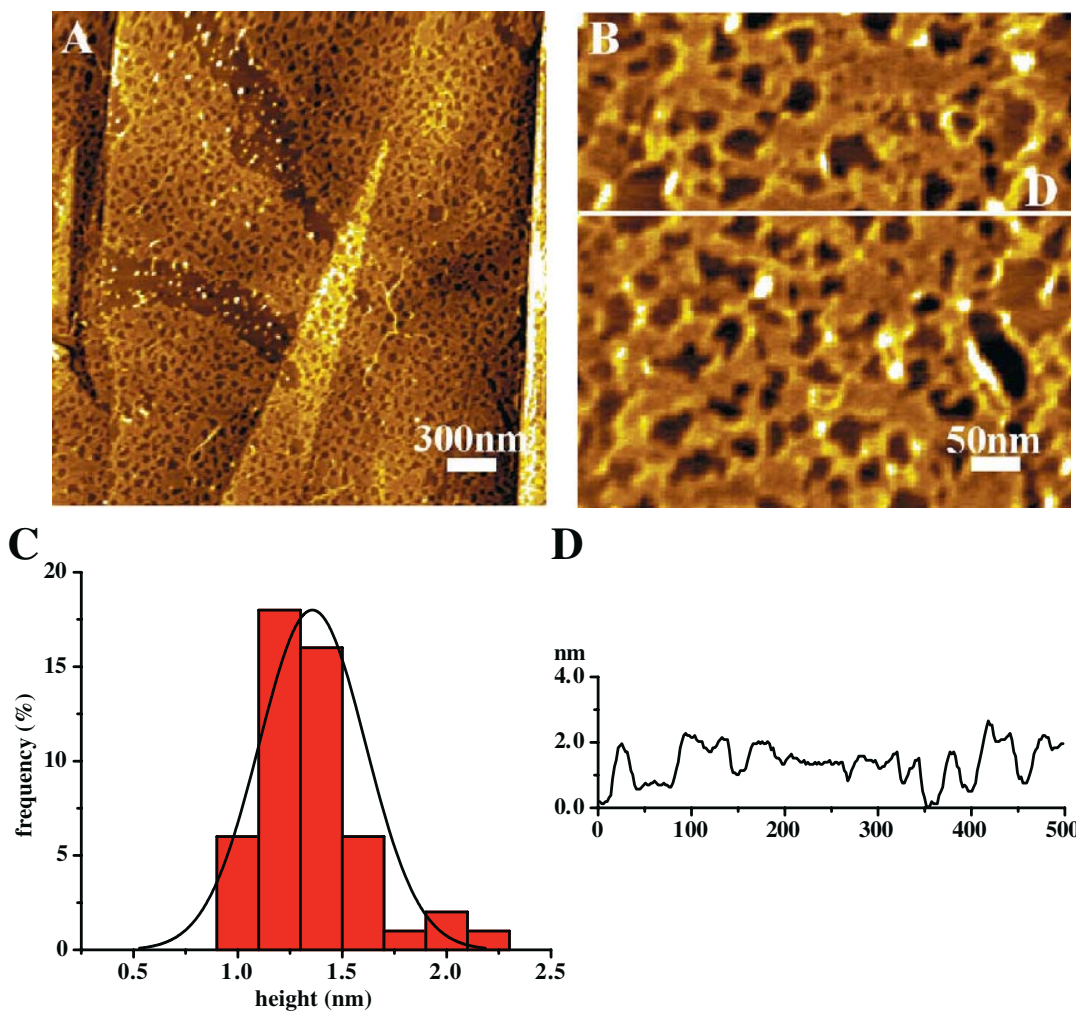

D

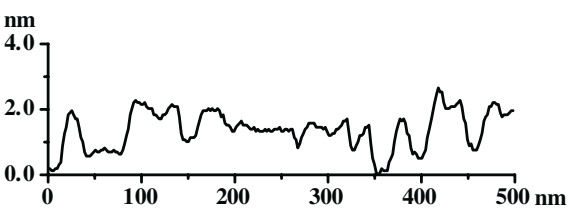

Fig. 5. (A, B) MAC Mode AFM topographical images in air of A8G2 molecules, immobilized onto HOPG by free adsorption during $3 \mathrm{~min}$, from $0.3 \mu \mathrm{M} A 8 \mathrm{G} 2$, in $\mathrm{pH}$ 4.5 0.1 M acetate buffer. (C) Histogram of the A8G2 film thickness generated with the values measured by section analyses inside the images. (D) Cross-section profile through white line in image (B). 

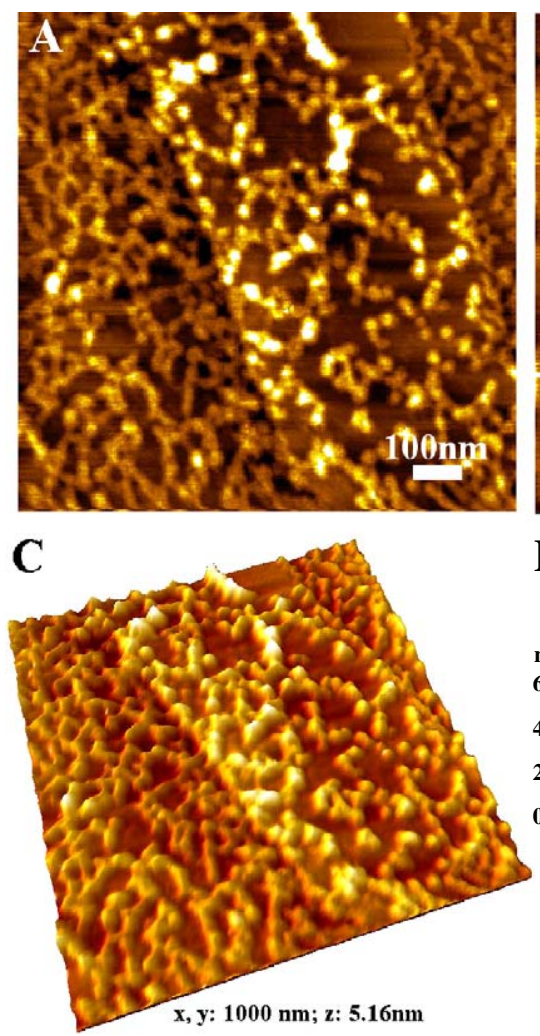

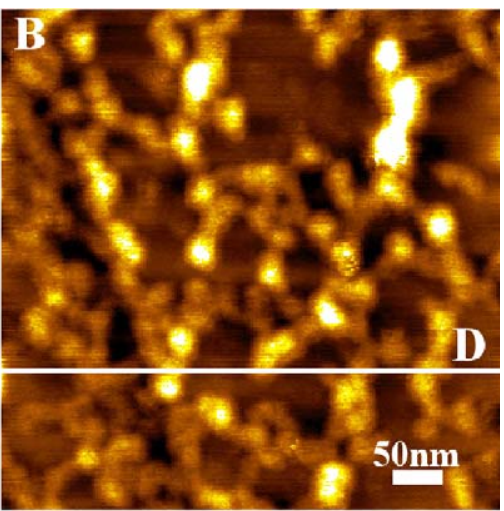

D

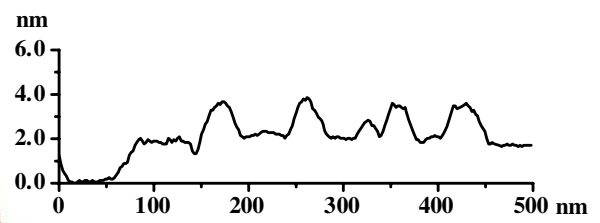

Fig. 6. (A, B) MAC Mode AFM topographical images in air of T8C2 molecules, immobilized onto HOPG by free adsorption during $3 \mathrm{~min}$, from $0.3 \mu \mathrm{M}$ T8C2, in pH 4.5 0.1 M acetate buffer. (C) Three-dimensional representation of image (A). (D) Cross-section profile through white line in image (B).

molecules demonstrated the predisposition to condense together in extensive thin lattices of $1.3 \pm 0.2 \mathrm{~nm}$ thickness, covering large areas of the substrate, Fig. 8B. A less densely packed network was imaged onto HOPG using MIX 2 solutions, Fig. $8 \mathrm{C}$, with the height of $1.3 \pm 0.4 \mathrm{~nm}$, Fig. $8 \mathrm{D}$. A few very high aggregates up to $5.0 \mathrm{~nm}$ were also measured.

\subsection{Interpretation of homo- and hetero-ODNs adsorption processes}

The surface of HOPG presents hydrophobic characteristics and the different adsorption morphologies obtained onto HOPG for the ODNs studied will be explained on the basis of the hydrophobicity of the ODN sequences.

The hydrophobicity of 10-mer ODN molecules results from the contribution of each constituent nucleotide. The hydrophobicity of each nucleotide is determined by the hydrophobicity of the aromatic base, Fig. 1, being proportional to its hydrophobic contact surface area. The bases, guanine, adenine, cytosine and thymine, due to their aromatic rings, adsorb onto HOPG and interaction of the $\pi$-electron system of the heteroaromatic rings of the bases with the HOPG hydrophobic surface is involved in the adsorption process.
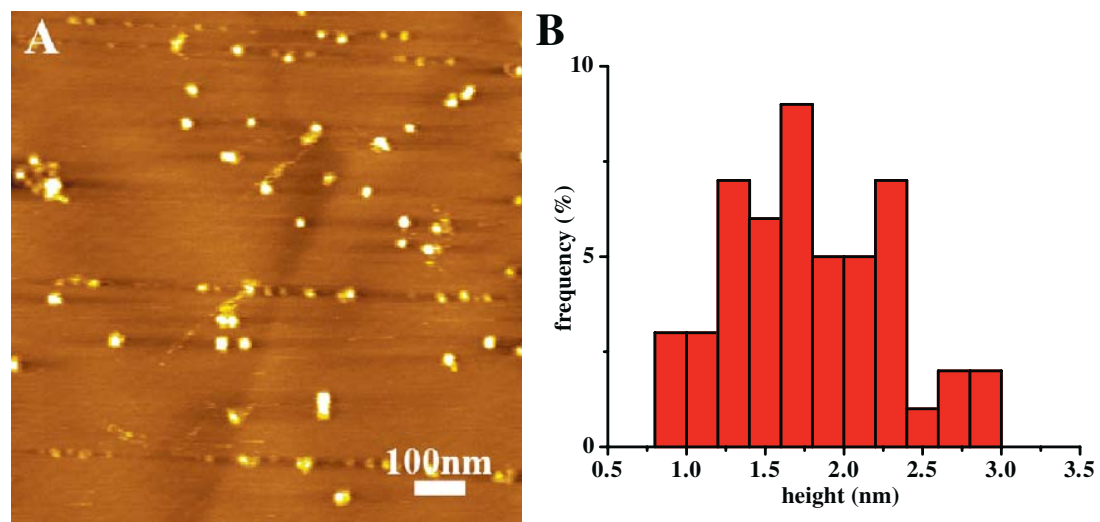

Fig. 7. (A) MAC Mode AFM topographical image in air of T6C4 molecules, immobilized onto HOPG by free adsorption during $3 \mathrm{~min}$, from $0.3 \mu \mathrm{M}$ T6C4, in pH 4.5 $0.1 \mathrm{M}$ acetate buffer. (B) Histogram of the T6C4 molecules heights generated with the values measured by section analyses inside the images. 


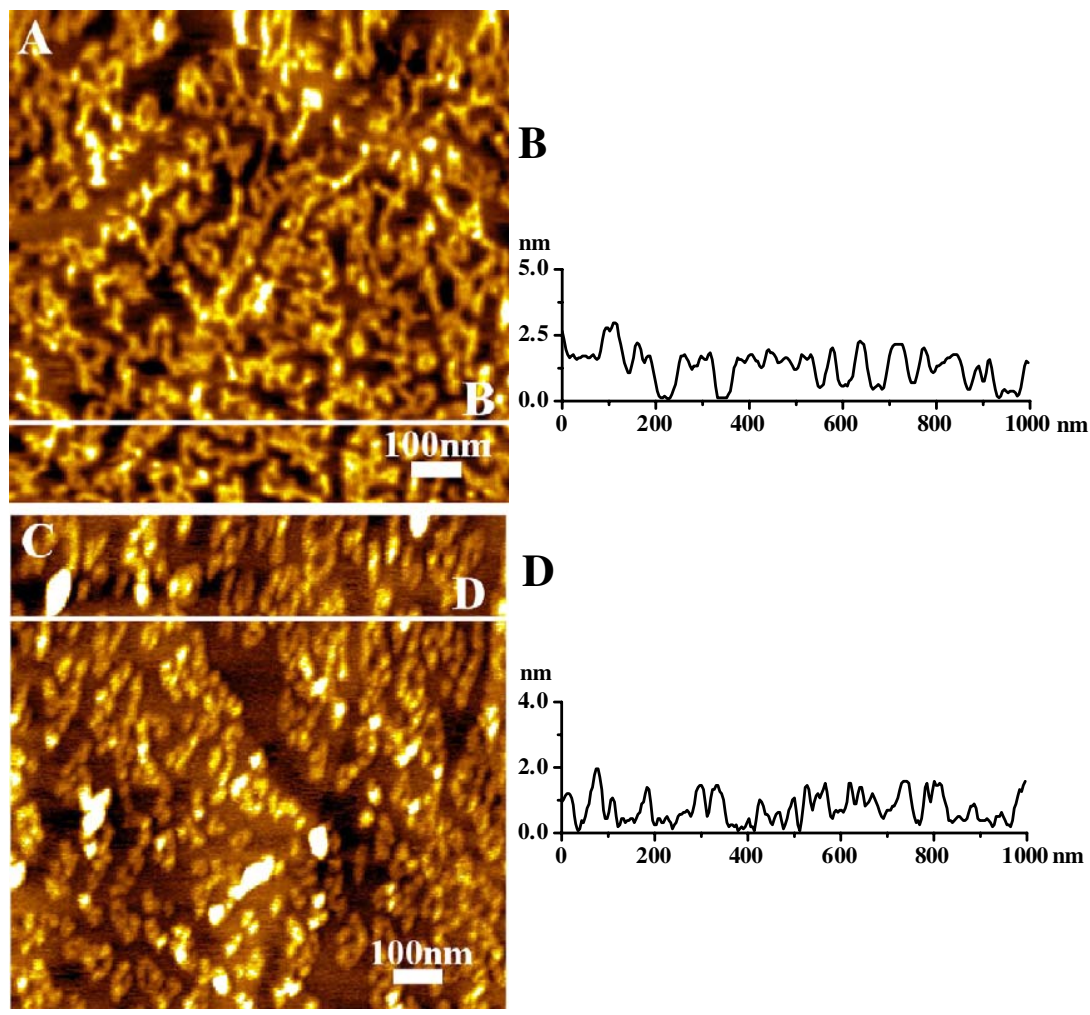

Fig. 8. (A, C) MAC Mode AFM topographical images in air of ODNs molecules, immobilized onto HOPG by free adsorption during 3 min, from $0.3 \mu \mathrm{M}$ (A) MIX 1 and (C) MIX 2, in pH 4.5 0.1 M acetate buffer. (B, D) Cross-section profiles through white line in images (A) and (C).

As described in the literature, the hydrophobicity of guanine is higher than the hydrophobicity of adenine, which is higher than that of thymine, in turn higher than the hydrophobicity of cytosine: $\mathrm{G}>\mathrm{A}>\mathrm{T}>\mathrm{C}[1]$.

The ODN molecular mass and the presence of secondary structures in the ODNs also influence the hydrophobic character of the molecules and consequently the adsorption onto HOPG.

Tables 1 and 2 summarize the morphological characteristics of the ODN sequences, in decreasing order of HOPG surface coverage during adsorption, obtained by AFM, Table 1 for the homo-ODNs and Table 2 for the hetero-ODNs.

The adsorption of homo-ODNs containing only adenines, guanines, thymines and cytosines, Table 1, will be compared. The degree of HOPG surface coverage by A10, T10 and C10 sequences followed the decrease of the ODN molecular mass and the hydrophobicity of the constituent bases $\mathrm{A}>\mathrm{T}>\mathrm{C}$.

The decrease in HOPG surface coverage with hetero-ODNs, Table 2, also follows the decrease of the hydrophobic character

Table 1

Characteristics of the homo-ODNs used in AFM experiments, presented in decreasing order of HOPG surface coverage during adsorption

\begin{tabular}{|c|c|c|c|c|}
\hline & ODN & $\begin{array}{l}\text { Sequence } \\
\left(5^{\prime} \rightarrow 3^{\prime}\right)\end{array}$ & $\begin{array}{l}M \\
\left(\mathrm{~g} \mathrm{~mol}^{-1}\right)\end{array}$ & $\begin{array}{l}\text { Secondary } \\
\text { structure }\end{array}$ \\
\hline \multirow{4}{*}{$\begin{array}{l}\text { HOPG } \\
\text { surface } \\
\text { coverage }\end{array}$} & A10 & A A A A A A A A A & 3137.5 & $\begin{array}{l}\text { Single+double } \\
\text { stranded }\end{array}$ \\
\hline & T10 & 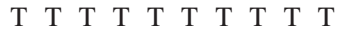 & 3047.4 & Single-stranded \\
\hline & G10 & $G G G G G G G G G$ & 3297.5 & G-quartets \\
\hline & $\mathrm{C} 10$ & $\mathrm{CCCHCHCHC}$ & 2897.4 & Double-stranded \\
\hline
\end{tabular}

of constituent bases and ODN molecular mass. The heteroODNs containing purine bases (adenine and guanine) in the chain generally presented a stronger adsorption onto HOPG when compared with the hetero-ODNs containing pyrimidine bases (thymine and cytosine). The different adsorption isotherms of the purine and pyrimidine bases at the graphitewater interface have been previously described in the literature [19], and the adsorption behaviour followed the hydrophobicity series of the bases: $G>A>T>C$, resulting from the existence of two aromatic rings in the case of the purines.

Therefore, the hydrophobicity of the bases which enter in the composition of the ODNs plays a very important role in the adsorption onto HOPG surfaces.

Considering MIX 1 and MIX 2 sequences that contain the same number of each base in the molecular chain, organized in a different order, interesting results were obtained. As observed in the AFM images, MIX 1 molecules, that contain guanine and

Table 2

Characteristics of the hetero-ODNs used in AFM experiments, presented in decreasing order of HOPG surface coverage during adsorption

\begin{tabular}{|c|c|c|c|c|}
\hline & ODN & $\begin{array}{l}\text { Sequence } \\
\left(5^{\prime} \rightarrow 3^{\prime}\right)\end{array}$ & $\begin{array}{l}M \\
\left(\mathrm{~g} \mathrm{~mol}^{-1}\right)\end{array}$ & $\begin{array}{l}\text { Secondary } \\
\text { structure }\end{array}$ \\
\hline $\begin{array}{l}\text { HOPG } \\
\text { surface } \\
\text { coverage }\end{array}$ & $\begin{array}{l}\text { A6G4 } \\
\text { A8G2 } \\
\text { MIX 1 } \\
\text { MIX } 2 \\
\text { T8C2 } \\
\text { T6C4 }\end{array}$ & $\begin{array}{llllllllll}\text { A } & \text { A } & \text { A } & \text { A } & G & G & \text { A } & G & \text { A } & G \\
\text { A } & \text { A } & \text { A } & G & \text { A } & \text { A } & \text { A } & \text { A } & \text { A } & G \\
\text { G } & \text { T } & \text { A } & G & \text { A } & \text { T } & \text { C } & \text { A } & \text { C } & \text { T } \\
\text { A } & G & \text { T } & G & \text { A } & \text { T } & \text { C } & \text { T } & \text { A } & \text { C } \\
\text { C } & \text { T } & \text { T } & \text { T } & \text { T } & \text { T } & \text { C } & \text { T } & \text { T } & \text { T } \\
\text { C } & \text { T } & \text { C } & \text { T } & \text { C } & \text { C } & \text { T } & \text { T } & \text { T } & \text { T }\end{array}$ & $\begin{array}{l}3201.5 \\
3169.5 \\
3094.4 \\
3094.4 \\
3017.4 \\
2987.4\end{array}$ & $\begin{array}{l}\text { Single-stranded } \\
\text { Single-stranded } \\
\text { Single-stranded } \\
\text { Single-stranded } \\
\text { Single-stranded } \\
\text { Double-stranded }\end{array}$ \\
\hline
\end{tabular}


thymine bases at the extremities of the chain showed an increased adsorption on the HOPG surface, Fig. 8A, when compared with MIX 2, that have adenine and cytosine at the ends, Fig. 8C. Consequently, it can be concluded that the bases existent at the strand extremities play the most important role in the adsorption process, due to an increased interaction and surface contact area with the HOPG surface.

However, the variations observed in the images cannot be interpreted only in terms of the hydrophobicity of the constituent bases.

The MAC Mode AFM images of the homo-ODNs reveal that the G10 molecules with high molecular mass and purine composition adsorb less, Fig. 2C, compared with T10 molecules with lower molecular mass and pyrimidine composition, Fig. $3 \mathrm{C}$. Also for the adsorption of G10 molecules, Fig. 2C, should be expected a higher surface coverage compared to the A10 molecules, Fig. 2A, due to the higher hydrophobic character of guanine bases. The hydrophobicities of T10 and C10 are very similar because the differences in molecular structure are very small, which therefore cannot explain such a big difference between the surface coverage of HOPG by T10 and C10 adsorbed molecules, Fig. 3.

The hetero-ODNs $\mathrm{T} 8 \mathrm{C} 2$ and $\mathrm{T} 6 \mathrm{C} 4$ also presented a very different adsorption pattern, Figs. 6 and 7, which cannot be explained only by the substitution of two thymines by two cytosines in the chain.

Another important factor that can influence the hydrophobicity of the ODN sequences and consequently the hydrophobic interaction with HOPG is the ODN secondary structure. The particularities observed in the homo- and hetero-ODNs adsorption behavior can be explained by the existence of different ODN secondary structures.

In DNA, the double-helical structure is stabilized by hydrogen bonds between the complementary bases adenine and thymine and between guanine and cytosine. However, the base-base recognition through hydrogen bonding is not restricted to interaction between complementary bases, and in special conditions, equal bases can also associate. DNA bases are uncharged at neutral $\mathrm{pH}$. Following a change of $\mathrm{pH}$, the bases can interact with another equal base, explaining the ability of the single-stranded homo-and hetero-ODN molecules to interact with each other and to form ordered and complex structures.

Depending on the $\mathrm{pH}$, in aqueous solutions, the A10 molecules exist in single- or double-helical form [1]. In neutral or alkaline solutions the single-helical species prevail, while at $\mathrm{pH}$ below 4 the adenines become protonated at the $\mathrm{N}_{1}$ group and the molecules aggregate to form double-helical structures with protonated base-pairs: $\mathrm{AH}^{+}-\mathrm{AH}^{+}$. At the $\mathrm{pH}$ 4.5 used in this study many adenine bases are protonated, and probably numerous molecules are preferentially organized, forming double-helical configurations. Several ODN molecules may exhibit rather complicated configurations, consisting of elements of both single and double-helical morphology.

In the case of G10 sequences, guanines have the ability to stack upon each other to form four-stranded structures with a guanine tetrad core. X-ray diffraction studies at neutral $\mathrm{pH}$ establish that poly $(\mathrm{G})$ homopolymers and their oligonucleotides form four-stranded nucleic acid structures with stacked guanine tetrads [1,20]. The guanine base has two $\mathrm{pK}$ values, corresponding to proton addition to $\mathrm{N}_{7}$ below $\mathrm{pH} 2.1$ and to proton loss from $\mathrm{N}_{1}$ above $\mathrm{pH}$ 9.2. Therefore guanine is neutral within a broad $\mathrm{pH}$ range, between $\mathrm{pH} 2.1$ and 9.2, including $\mathrm{pH}$ value of 4.5 used in the AFM study, which favors the stabilization of four-stranded structures. Thus, it can be considered that at $\mathrm{pH} 4.5$ many G10 molecules form G-quartets morphology in solution.

In aqueous solutions, at $\mathrm{pH} 4.5$, the $\mathrm{pK}$ of cytidine, the mononucleotides $\mathrm{C} 10$ form a double helix with parallel chains and hemiprotonated base-pairs $\mathrm{CH}^{+}-\mathrm{C}$ [1].

The pyrimidinic T6C4 sequences have a high number of (CT) repeats, which can influence the secondary structure of the molecule. NMR studies of ODNs containing alternating (CT) pyrimidine base sequences demonstrated that their conformations are strongly $\mathrm{pH}$ dependent [21,22] and depending on the $\mathrm{pH}$, at least three major conformational species appear. At a neutral, $\mathrm{pH}$ of 7 , the molecules adopt an antiparallel-stranded duplex with only $\mathrm{C}-\mathrm{T}$ base pairing and under highly acidic conditions, $\mathrm{pH} 3$, adopt an antiparallelstranded duplex composed only of $\mathrm{CH}^{+}-\mathrm{T}$ base pairs. At intermediate $\mathrm{pH}$ values, such as $\mathrm{pH} 4.5$ as used here, multiple conformational species coexist, with antiparallel-stranded duplexes of $\mathrm{C}-\mathrm{T}$ and $\mathrm{CH}^{+}-\mathrm{T}$ base pairs, and also, possibly parallel-stranded duplexes with $\mathrm{CH}^{+}-\mathrm{C}$ base pairs. Consequently, the $\mathrm{T} 6 \mathrm{C} 4$ molecules are expected to form complex and compact molecular structures, with the pyrimidine bases packed inside the double helix.

In any case, the adsorption of small 10-mer ODNs seems to be strongly influenced by modifications to the ODN secondary structure that determines the exposure of the hydrophobic bases and reduces the exposed hydrophobic surface area of the molecules.

As observed in Table 1, for the same solution concentration, the homo-ODNs A10 and T10 containing single-stranded species interact and adsorb strongly onto the HOPG surface, when compared with double-helical C10 and quadruple G10 ODNs. The A10 and G10 sequences still adsorb onto HOPG, due to the superior hydrophobicity of the purinic bases at the chain extremities, while the adsorption of pyrimidinic C10 sequences was almost completed inhibited.

In the case of hetero-ODNs, Table 2, all single-stranded sequences presented an increased adsorption onto HOPG compared to the T6C4 double-strands.

The interaction of single-stranded ODNs with the HOPG substrate is facilitated, because the single-stranded molecules have the bases exposed to the solution and are free to undergo hydrophobic interactions with the hydrophobic HOPG surface.

In all AFM experiments it was observed that the singlestranded molecular films had larger heights than the doublestranded ODNs, which suggests that the single-stranded ODNs tend to fold back on themselves and interact more easily with each other 
The ODNs with double-helical and quartet morphology formed at $\mathrm{pH} 4.5$ are hydrophilic and the negatively charged phosphate groups are on the exterior of the molecules. The increased number of polar phosphate groups adds a negative contribution to the net hydrophobicity of the molecules, decreasing the hydrophobic interaction with the surface. Since the bases from the interior of the molecules are protected by the sugar-phosphate backbones, the double or quadruple ODNs adsorb on the HOPG surface only through hydrophobic interactions between the bases at the extremities of the ODN chain and the HOPG.

On the other hand, due to the formation of complex secondary structures, the number of ODNs in quadruple and double-helical configurations is inferior to the number of ODNs that remain single-stranded. Consequently the number of quadruple and double-helical structures adsorbed on the HOPG surface is reduced.

It can be concluded that the hydrophobic interactions of the ODN molecules with the surface represent the main adsorption mechanism, although other effects such as electrostatic and Van der Waals interactions may contribute as well to the adsorption process.

The AFM results clarify the adsorption mechanism of ODN molecules and have strong implications in the development of DNA-electrochemical biosensors. The hybridization approach at a DNA-electrochemical biosensor consists in the immobilization of the ODN probe sequence on the electrode surface and the hybridization of the probe with the complementary ODN target, present in the solution.

The ODNs used in this study correspond to pairs of complementary sequences: A10 with T10, G10 with C10, A6G4 with T6C4, A8G2 with T8C2 and MIX 1 with MIX 2. However, as observed in the AFM images, each of these complementary ODN structures always presented a very different adsorption morphology and coverage onto the HOPG surface. This aspect must be carefully considered when choosing one sequence as a probe to be immobilized on the electrode surface and the other as a target for hybridization.

The electrode surface coverage, orientation and packing of the adsorbed ODN probe layer are very important. Ideally, the probe ODNs must be connected with the electrode surface at one point only, oriented with the sequences perpendicular to the electrode surface in such a way that the target sequence will have a total access to the immobilized probe, the molecules of which are sufficiently separated one from another to be able to enable hybridization. Besides this, they must present a uniform coverage of the surface in order to minimize non-specific adsorption of target molecules on the uncovered areas of the electrode. Additionally, the target ODNs should be chosen so as to have a reduced adsorption on the carbon electrode surface, in order to minimize non-specific adsorption. Consequently, the sequences dynamic secondary structure always has to be considered.

\section{Conclusions}

In order to understand the mechanism of interaction of nucleic acids with the HOPG surface, the adsorption process of synthetic homo- and hetero-ODN molecules was studied at room temperature in air, using MAC Mode AFM. Several factors affecting the degree of HOPG surface coverage were analyzed.

The 10-mer ODN molecules adsorbed spontaneously on the HOPG surface, through hydrophobic interactions with the hydrophobic surface and the adsorption is strongly influenced by the base type in the ODN sequence. The importance of the type of base at the sequence extremities on the adsorption process was evaluated. The differences in HOPG surface coverage obtained with ODN molecules with mixed base composition and the same number of each base, in different orders, demonstrated that the type of base at the molecule extremities plays the most important role in the adsorption process.

The molecular mass and the presence of secondary structures in the ODNs influence the hydrophobic character of the molecules and consequently the adsorption onto HOPG. Single-stranded ODN molecules interacted and adsorbed strongly onto HOPG, since the bases are more exposed and free to undergo hydrophobic interactions with the HOPG surface. The formation of double-helical or more complex structures reduced the interaction of the ODN molecules with the surface, because the molecules had the bases protected in the interior of the secondary structure.

The AFM results clearly show the importance of characterizing and understanding the adsorption process of small ODNs on carbon electrode surface, and evaluation of all the factors influencing the correct manufacture of DNA electrochemical biosensors for hybridization detection.

\section{Acknowledgments}

Financial support from Fundação para a Ciência Tecnologia (FCT) Post-Doc Grant SFRH /BPD/14425/2003 (A.-M. C.P.), POCTI (co-financed by the European Community Fund FEDER), ICEMS (Research Unit 103), and European Project HPRN-CT-2002-00186 are gratefully acknowledged.

\section{References}

[1] W. Saenger, Principles of nucleic acid structure, in: Ch.R. Cantor (Ed.), Springer Advanced Texts in Chemistry, Springer-Verlag, New York, 1984

[2] R.F. Service, Biology offers nanotechs a helping hand, Science 298 (2002) 2322-2323.

[3] H. Yan, X. Zhang, Z. Shen, N.C. Seeman, A robust DNA mechanical device controlled by hybridization topology, Nature 415 (2002) 62-65.

[4] I. Willner, Biomaterials for sensors, fuel cells, and circuitry, Science 298 (2002) 2407-2408.

[5] A.M. Oliveira Brett, S.H.P. Serrano, J.A.P. Piedade, Electrochemistry of DNA, comprehensive chemical kinetics, in: R.G. Compton, G. Hancock (Eds.), Applications of Kinetic Modeling, vol. 37, Elsevier, Oxford, UK, 1999, pp. 91-119, chapter 3.

[6] A.M. Oliveira Brett, DNA-based biosensors, in: L. Gorton (Ed.), Comprehensive Analytical Chemistry, Biosensors and Modern Specific Analytical Techniques, vol. 44, 2005, pp. 179-208.

[7] A.M. Oliveira Brett, M. Vivan, I.R. Fernandes, J.A.P. Piedade, Electrochemical detection of in situ adriamycin oxidative damage to DNA, Talanta 56 (2002) 959-970. 
[8] E. Palecek, M. Fojta, M. Tomschik, J. Wang, Electrochemical biosensors for DNA hybridization and DNA damage, Biosens. Bioelectron. 13 (1998) 621-628.

[9] M. Mascini, I. Palchetti, G. Marrazza, DNA electrochemical biosensors, Fresenius' J. Anal. Chem. 369 (2001) 15-22.

[10] R.F. Service, Getting a feel for genetic variations, Science 289 (2000) 27-28.

[11] D.R. Meldrum, Sequencing genomes and beyond, Science 292 (2001) 515-517.

[12] H.G. Hansma, I. Revenko, K. Kim, D.E. Laney, Atomic force microscopy of long and short double-stranded, single-stranded and triple-stranded nucleic acids, Nucleic Acids Res. 24 (1996) 713-720.

[13] A.M. Oliveira Brett, A.-M. Chiorcea, Effect of $\mathrm{pH}$ and applied potential on the adsorption of DNA on highly oriented pyrolytic graphite electrodes. Atomic force microscopy surface characterisation, Electrochem. Commun. 5 (2003) 178-183.

[14] A.M. Oliveira Brett, A.-M. Chiorcea, Atomic force microscopy of DNA immobilized onto a highly oriented pyrolytic graphite electrode surface, Langmuir 19 (2003) 3830-3839.

[15] A.M. Oliveira Brett, A.-M. Chiorcea, DNA imaged on a HOPG electrode surface by AFM with controlled potential, Bioelectrochemistry 1-2 (2005) $117-124$.
[16] A.M. Oliveira Brett, A.-M. Chiorcea, Atomic force microscopy characterization of an electrochemical DNA-biosensor, Bioelectrochemistry 63 (2003) 229-232.

[17] F. Zenhausern, M. Adrian, R. Emch, M. Taborelli, M. Jobin, P. Descouts, Scanning force microscopy and cryo-electron microscopy of tobacco mosaic virus as a test specimen, Ultramicroscopy 42-44 (1992) $1168-1172$

[18] C. Bustamante, J. Vesenka, C.L. Tang, W. Rees, M. Guthold, R. Keller, Circular DNA molecules imaged in air by scanning force microscopy, Biochemistry 31 (1992) 22-26.

[19] S.J. Sowerby, C.A. Cohn, W.M. Heckl, N.G. Holm, Differential adsorption of nucleic acid bases: relevance to the origin of life, Proc. Natl. Sci. U. S. A. 98 (2001) 820-822.

[20] T. Simonsson, G-Quadruplex DNA structures - variation of a theme, Biol. Chem. 382 (2001) 621-628.

[21] T.N. Jaishree, A.H. Wang, Conformations of the alternating (C-T)n sequence under neutral and low pH, FEBS Lett. 337 (1994) 139-144.

[22] T.N. Jaishree, A.H. Wang, NMR studies of $\mathrm{pH}$-dependent conformational polymorphism of alternating $(\mathrm{C}-\mathrm{T}) \mathrm{n}$ sequences, Nucleic Acids Res. 21 (1993) 3839-3844. 